\section{NOUVELL $\varepsilon$}

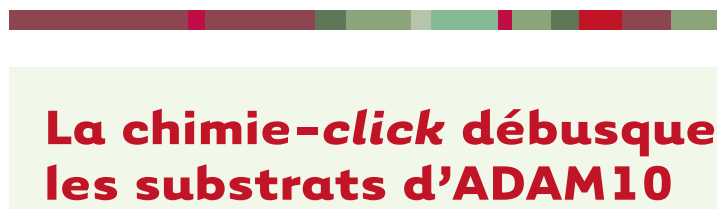

Mirca Saurty ${ }^{1}$, Romain Sanson ${ }^{1}$, Rania Amrane ${ }^{1}$, Eric Rubinstein ${ }^{2,3}$
${ }^{1}$ Ml Biologie Santé, Université Paris-Saclay, 91405, Orsay;

2 Inserm U935, 94807 Villejuif, France ;

${ }^{3}$ Université Paris-Sud, Université Paris-

Saclay, Institut André Lwoff, 94807 Villejuif, France.

mirca.saurty@u-psud.fr

romain.sanson@u-psud.fr

rania.amrane@u-psud.fr

eric.rubinstein@inserm.fr
> Les protéines de la famille ADAM ( $a$ desintegrin and metalloprotease) sont des métalloprotéases membranaires dont le rôle est de cliver diverses protéines membranaires libérant ainsi leur partie extracellulaire, ou ectodomaine. Ces protéases sont notamment responsables, via ce mécanisme, de la sécrétion de cytokines et de facteurs de croissance synthétisés sous forme de précurseurs membranaires, par exemple le tumour necrosis factor alpha (TNF $\alpha$ ) ou les ligands du récepteur de I'EGF (epidermal growth factor) [1]. ADAM10 en particulier joue un rôle essentiel dans la signalisation en aval des récepteurs Notch, une voie de signalisation majeure au cours du développement et qui contrôle l'homéostasie de nombreux tissus. Le clivage du domaine extracellulaire des récepteurs Notch par ADAM10 permet en effet un deuxième clivage transmembranaire par la $\gamma$-sécrétase, permettant alors la libération du domaine cytosolique de Notch, qui peut exercer son action de cofacteur de transcription dans le noyau [1].

Un autre substrat majeur d'ADAM10 est la protéine transmembranaire APP (amyloid precursor protein), le précurseur du peptide amyloïde bêta $(A \beta)$ impliqué dans la maladie d'Alzheimer [1]. Son clivage par ADAM10 empêche la formation du peptide $A \beta$, ce qui a conduit certains chercheurs à proposer la stimulation de

Cette Nouvelle fait partie d'une série de 15 Nouvelles rédigées par les étudiants du Master «Biologie Santé » de l'université Paris-Saclay qui paraîtront dans les numéros $6-7,8-9$ et 10 (2016) de médecine/sciences. l'activité d'ADAM10 comme une approche thérapeutique possible dans la maladie d'Alzheimer [2].

L'importance physiologique d'ADAM10 est soulignée par le fait que les souris knock-out pour le gène correspondant meurent au stade embryonnaire. Lorsque le gène codant ADAM10 est invalidé spécifiquement dans les neurones après la naissance, les souris présentent des épisodes d'épilepsie, des déficits d'apprentissage et des altérations des structures postsynaptiques [3]. Cependant, les substrats connus d'ADAM10 n'expliquent qu'en partie ces phénotypes, incitant Kuhn et ses collègues à chercher d'autres substrats d'ADAM10 dans le système nerveux central [4].

\section{Quand la chimie-click s'y colle}

Les auteurs ont analysé par spectrométrie de masse quantitative le répertoire de protéines sécrétées par des neurones en culture selon qu'ils sont déficients ou non en ADAM10. Pour contourner l'obstacle de la létalité embryonnaire des animaux knock-out pour ADAM10, ils ont utilisé des neurones issus de souris dont le gène $A D A M 10$ est flanqué de sites lox, et ont induit la délétion d'ADAM10 in vitro en transduisant la recombinase $\mathrm{Cre}^{\mathrm{l}}$ via des lentivirus. Un second obstacle lors de l'analyse du sécrétome est la présence de protéines issues du sérum et de protéines cytosoliques relâchées par les cellules mortes. Cette

${ }^{1}$ Le système de recombinaison Cre-lox utilise l'enzyme recombinase Cre, une tyrosine recombinase issue du bactériophage $\mathrm{Pl}$, afin de cibler des séquences loxP (également issues du bactériophage $\mathrm{Pl}$ ), permettant ainsi d'activer, réprimer, voire même échanger, les gènes situés entre les séquences lox. difficulté a été contournée en utilisant la chimie «click» (voir Encadré 1) : les cellules ont été incubées avec un dérivé du mannosamine qui réalise un marquage métabolique des protéines glycosylées. Ce dérivé (ManNAZ) porte une fonction azide qui permet son couplage par chimie-click à d'autres molécules portant une fonction alkyne ou dibenzocyclooctyl (DBCO). Ainsi, seules les glycoprotéines nouvellement synthétisées sont effectivement marquées ; elles peuvent ainsi être couplées à la biotine par chimie-click, ce qui permet leur purification avec de la streptavidine.

\section{De nouveaux substrats neuronaux} d'ADAM10 identifiés !

L'approche non biaisée adoptée par les auteurs a permis d'identifier 313 glycoprotéines d'origine cellulaire dans le milieu des neurones en culture, dont 200 sont des protéines membranaires. Près de la moitié d'entre elles (principalement des protéines membranaires de type I) sont présentes en quantité significativement diminuée en l'absence d'ADAM10, et représentent donc des substrats potentiels de la protéase.

La grande majorité des protéines dont la libération dépend d'ADAMIO sont des molécules d'adhérence et des récepteurs. Parmi ces protéines, la seule cytokine identifiée est la chimiokine CX3CLl ( $C-X 3-C$ motif chemokine ligand 1 ) ou fractalkine. Cette chimiokine, substrat connu d'ADAM10, pourrait avoir un rôle neuroprotecteur en diminuant l'activation des cellules microgliales [7]. Il est cependant possible que d'autres cytokines n'aient pas été identifiées dans 


\section{La chimie-click en biologie}

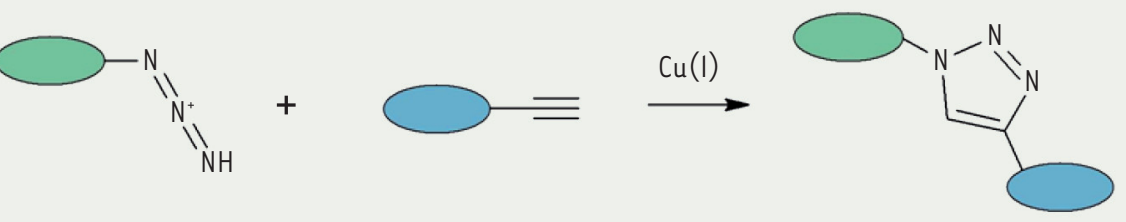

Azide

Alcyne

Triazole
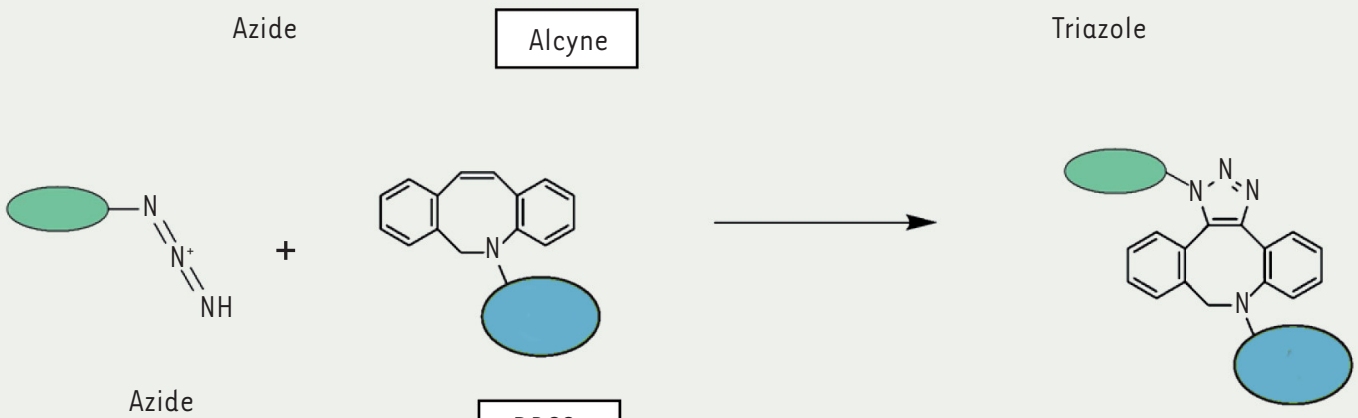

DBCO

Le concept de chimie-click a été introduit en 2001 par Sharpless [5]. II définit un groupe de réactions chimiques quasi parfaites, modulables, simples à mettre en œuvre et se produisant en absence de solvant. Si la plus connue est la réaction de cycloaddition azoture-alcyne catalysée par le cuivre (CuAAC), des approches sans cuivre ont été développées, notamment celles utilisant le DBCO (dibenzocyclooctyl) employé par Kuhn et al. [4]. Ces réactions sont d’une grande sélectivité, permettant de coupler divers types de molécules fonctionnalisées sans interférer avec l'environnement biologique, et ont donc trouvé de nombreuses applications en biologie. Elles ont par exemple été utilisées pour le développement de nouvelles drogues, le marquage de biomolécules, ou différents types de marquages métaboliques (protéines, ADN, lipides) [6].

cette étude pour laquelle les auteurs ont fait le choix de s'intéresser aux protéines de plus de 30 kDa.

Un premier grand groupe fonctionnel de protéines membranaires dont le clivage est dépendant pour une large part d'ADAMIO est celui de protéines impliquées dans la formation et la fonction des synapses. Elles peuvent être localisées sur les membranes pré- ou post-synaptiques. II s'agit parfois de molécules capables d'interagir en trans. C'est notamment le cas de certaines PTPR (protein tyrosine phosphatase, receptor type), et de leurs ligands sur la membrane postsynaptique, les protéines SLITRK (SLIT and NTRK-like) [8]. Plusieurs neuroligines $^{2}$, présentes sur la membrane post-synaptique, sont également libérées de façon dépendante d'ADAMIO [9]. En revanche, les neurexines aux-

\footnotetext{
${ }^{2}$ Les mutations des neuroligines 3 et 4 ont notamment été impliquées dans une forme d'autisme touchant la jonction synaptique.
}

quelles elles se lient sont aussi sécrétées, mais de manière indépendante de cette protéase.

Un autre groupe de cibles potentielles d'ADAM10 joue un rôle dans le ciblage axonal. II s'agit notamment de la néogenine (NEOl), récepteur de molécules de guidage axonal dont la nétrine [10], et de plusieurs membres de la famille des molécules d'adhérence Ll [11]. Les auteurs démontrent par ailleurs, pour renforcer l'hypothèse d'un rôle d'ADAM10 dans le ciblage axonal, des défauts de ce ciblage dans le bulbe olfactif et l'hippocampe chez les animaux knock-out pour ADAM10.

Les auteurs ont également étudié par la même approche le profil d'expression des protéines membranaires. Dans la plupart des cas, l'inhibition ou la diminution de la sécrétion des protéines étudiées provoquée par l'absence d'ADAM10 ne s'accompagnent pas d'un changement de leur expression au niveau cellulaire. Ceci suggère que le clivage de ces molécules par ADAM10 est régulé spa- tialement ou temporellement de façon très précise.

L'effet de l'absence d'ADAM10 sur la libération des protéines identifiées est très variable, l'inhibition pouvant varier de $30 \%$ à $95 \%$. Ceci indique que certaines de ces protéines sont clivées par d'autres protéases; il peut s'agir, dans certains cas mais pas dans tous, de BACE-1, dont l'activité est nécessaire à la production du peptide $\beta$-amyloïde [2]. Par ailleurs, les conséquences de l'absence d'ADAM10 peuvent être indirectes, la protéine incriminée n'étant pas une cible directe d'ADAM10. Par exemple, l'absence d'ADAMIO entraîne une diminution du clivage de la métalloprotéase MMP17 ancrée à la membrane; ce qui pourrait retentir en retour sur le clivage des substrats de MMP17. Enfin, l'absence d'ADAM10 conduit à des modifications cellulaires allant au-delà du clivage de protéines membranaires, comme le montrent la modification de la sécrétion de protéines sécrétées via la voie 


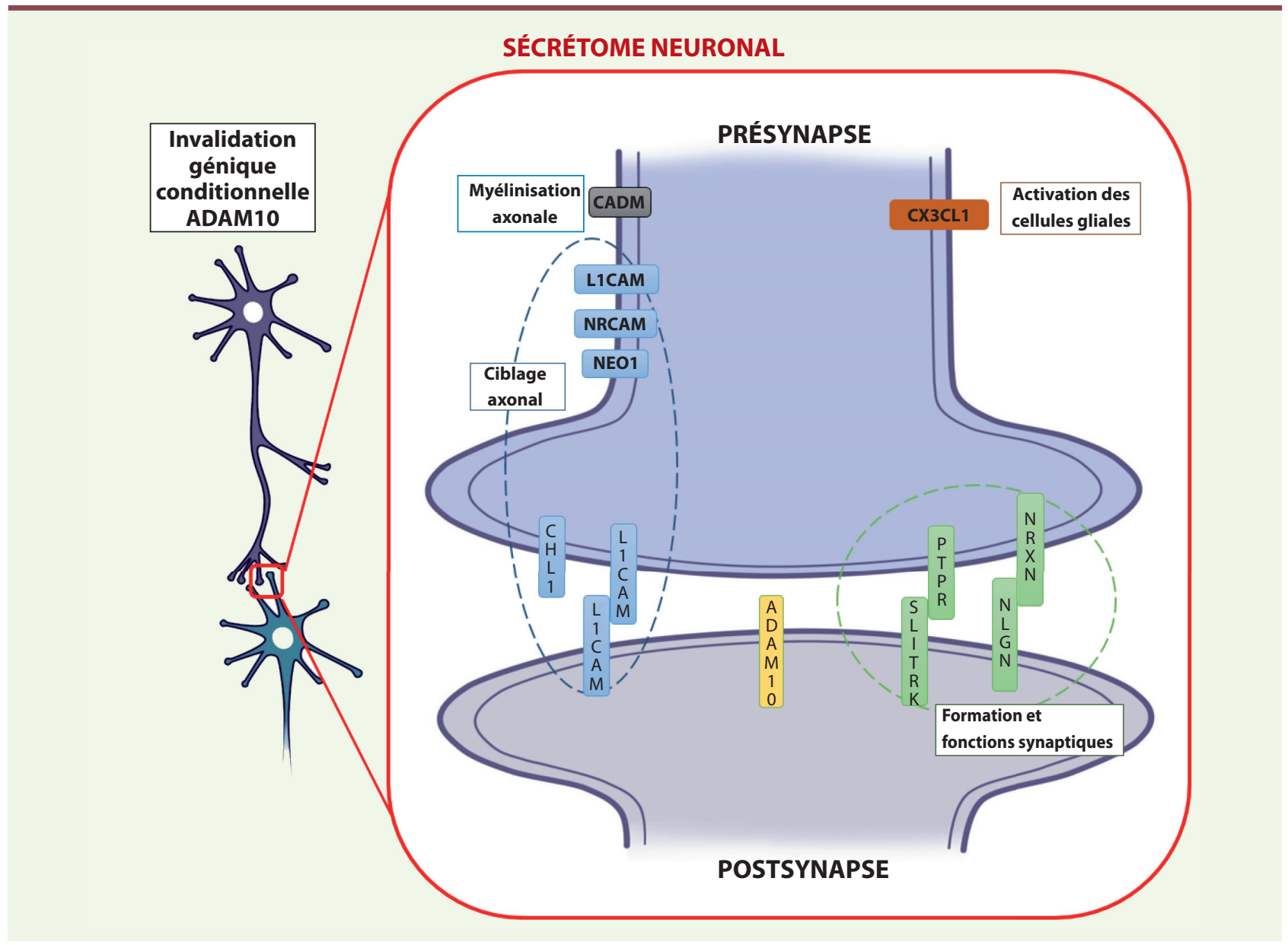

Figure 1. Vue globale de la localisation subcellulaire et des fonctions des substrats neuronaux d'ADAM10. Les substrats d'ADAM10 peuvent être regroupés selon leurs fonctions. On retrouve ainsi des substrats impliqués dans: (1) la formation et les fonctions synaptique (PTPR, SLITRK, NLGN, NRXN), (2) le ciblage axonal (NRCAM, NEOl, LI, CHLI), (3) la myélinisation axonale (CADM) et (4) l'activation des cellules gliales (CX3CLI). ADAM10 : a desintegrin and metalloprotease 10 ; PTPR : protein tyrosine phosphatase, receptor type; SLITRK : SLIT and NTRK-like ; NLGN : neuroligine; NRXN : neurexin ; NRCAM : neuronal cell adhesion molecule ; NE01 : neogenin ; Ll : CAM (neural cell adhesion molecule L1 precursor) ; CHL1 : neural cell adhesion molecule L1-like protein; CADM : cell adhesion molecule; CX3CL1 : chemokine (C-X3-C motif) ligand 1 ou fractalkine.

classique (qui ne fait pas intervenir un clivage protéolytique), et l'augmentation de l'expression de la sous-unité grial du récepteur du glutamate AMPA.

\section{Perspectives}

En conclusion, cette analyse - la plus exhaustive à ce jour de la fraction du sécrétome modulée par ADAMI0 constitue un bel exemple des possibilités qu'offre la chimie-click aux biologistes. Elle montre que de nombreux substrats de l'enzyme sont impliqués dans la formation et les fonctions synaptiques, ainsi que dans le ciblage axonal, suggérant un rôle majeur d'ADAMl0 dans ces fonctions, et expliquant ainsi une partie des phénotypes des animaux invalidés génétiquement pour ADAM10. La stimulation de l'activité d'ADAM10 a été proposée comme approche thérapeutique dans la maladie d'Alzheimer. La connaissance des substrats d'ADAM10 permettra une meilleure compréhension des effets secondaires potentiellement liés à cette approche. $\diamond$

In search of new substrates of ADAM10

\section{LIENS D'INTÉRÊT}

Les auteurs déclarent n'avoir aucun lien d'intérêt concernant les données publiées dans cet article.

\section{RÉFÉRENCES}

1. Reiss K, Saftig P. The a disintegrin and metalloprotease (ADAM) family of sheddases: physiological and cellular functions. Semin Cell Dev Biol 2009 ; 2 : 126-37.

2. Endres K, Fahrenholz F. Upregulation of the alphasecretase ADAM10: risk or reason for hope? FEBS J $2010 ; 277: 1585-96$.

3. Saftig $P$, Lichtenthaler SF. The alpha secretase ADAM10: a metalloprotease with multiple functions in the brain. Prog Neurobiol $2015 ; 13: 1-20$.

4. Kuhn P, Colombo A, Schusser B, et al. Systematic substrate identification indicates a central role for the metalloprotease ADAM10 in axon targeting and synapse function. ELIFE 2016 ; 5 : e12748.

5. Kolb HC, Finn MG, Sharpless KB. Click chemistry: diverse chemical function from a few good reaction. Angew Chem Int Ed Engl 2001 ; 40 : 2004-21.

6. Best M. Click chemistry and bioorthogonal reactions: unprecedented selectivity in the labeling of biological molecules. Biochemistry 2009; $48: 6571-84$. 


\section{RÉFÉRENCES}

7. Morganti JM, Nash KR, Grimmig BA, et al. The soluble isoform of $\mathrm{CX} 3 \mathrm{CL} 1$ is necessary for neuroprotection in a mouse model of Parkinson's disease. J Neurosci $2012 ; 32$ : 14592-601.
8. Lee R. Protein tyrosine phosphatase PTPRT as a regulator of synaptic formation and neuronal development. Bioch Mol Biol Rep 2015 ; 48 : 249-55

9. Krueger D, Tuffy L, Papadopoulos T, et al. The role of neurexins and neuroligins in the formation, maturation, and function of vertebrate synapses. Curr Opin Neurobiol $2012 ; 22$ : 412-22.
10. De Vries M, Cooper HM. Emerging roles for neogenin and its ligands in CNS development. J Neurochem $2008 ; 106: 1483-92$.

11. Hortsch M, Nagaraj K, Mualla R. The Ll family of cell adhesion molecules: a sickening number of mutations and protein functions. Adv Neurobiol $2014 ; 8$ : 195-229.

\section{NOUVELLE}

\section{Régénération hépatique}

\section{Deux pièces du puzzle réunies}

Anthony Lambert ${ }^{1}$, Boris Julien ${ }^{2}$

\author{
${ }^{1}$ Ml Biologie Santé, Université Paris-Saclay, 91405 Orsay, \\ France ; \\ ${ }^{2}$ Inserm U-1174, Université Paris-Sud, 91405 Orsay, France. \\ anthony.lambert@u-psud.fr \\ boris.julien@u-psud.fr
}

\section{La régénération hépatique :}

un processus complexe avec encore de nombreuses zones d'ombre

Le foie, situé à une position anatomique stratégique à l'interface entre le tractus digestif et la circulation sanguine générale, assure un très grand nombre de fonctions métaboliques et immunitaires de notre organisme. Ces fonctions l'exposent à des agressions variées et expliqueraient en partie pourquoi, chez les mammifères, le foie a conservé au cours de l'évolution une exceptionnelle capacité de régénération. Cette propriété du foie est utilisée dans la prise en charge de certaines pathologies hépatiques. Ainsi, elle permet d'envisager l'hépatectomie partielle avec peu de complications à long terme, notamment dans le contexte d'une résection tumorale. De plus, la transplantation d'un greffon hépatique issu d'un donneur vivant est également possible pour remplacer le foie non fonctionnel d'un patient, puisque le foie rémanent du donneur ainsi que le greffon chez le receveur régénéreront pour restituer un foie de taille normale.

Il est déjà bien établi que la régénération mobilise un très grand nombre d'acteurs cellulaires et moléculaires, non seulement localement dans le foie, mais aussi dans tout l'organisme. Ces interactions vont réguler la progression du cycle des différents types cellulaires du foie ainsi que la survie des hépatocytes exposés à de nombreux stress lors de la régénération. Cependant, la complexité de ce processus est telle que beaucoup de zones d'ombre subsistent quant à la nature des signaux intercellulaires impliqués, leur source et leurs cibles [1].

Le travail de Kudira et al. [2] - dont nous analysons ici les résultats - améliore notre compréhension du réseau d'interactions régulant la régénération. $\varepsilon n$ effet, cette équipe avait déjà montré que la signalisation purinergique (l'adénosine triphosphate [ATP] extracellulaire, ses produits de dégradation et leurs récepteurs) contrôlait les propriétés pro-régénératrices des cellules natural killer (NK) après résection hépatique, sans pouvoir en déterminer les mécanismes précis [3]. Dans cette nouvelle étude [2], les auteurs explicitent ce mécanisme et montrent que la signalisation purinergique stimule la production d'interleukine 22 (IL-22) - une cytokine hépatoprotectrice - par les innate lymphoid cells (ILC), famille de cellules de la lignée lymphoïde impliquées dans la réponse immunitaire innée et à laquelle appartiennent les cellules NK $([4])(\rightarrow)$. $(\rightarrow)$ Voir la Synthèse de M. Cherrier récemment consacrée à ce thème, $m / s n^{\circ} 3$, mars 2014, page 280
L'IL-22 protège le parenchyme après hépatectomie partielle Les signaux émis par les cellules de l'immunité innée jouent un rôle central dans la régulation fine du déclenchement, de la progression et de la terminaison du processus de régénération. Cependant, ces cellules peuvent avoir des fonctions ambivalentes sur la progression de la réparation tissulaire selon les messages qu'elles reçoivent. Par exemple, les macrophages favorisent la régénération en produisant de I'IL-6 [1], mais ils peuvent également l'inhiber via la sécrétion d'IL-10 [5]. II est donc important d'étudier les médiateurs libérés par les cellules de l'immunité innée ainsi que les conditions environnementales qui déterminent leur production.

L'IL-22 est sécrétée par les cellules de l'immunité et exerce des propriétés hépatoprotectrices [6]. Kudira et al. [2] se sont intéressés dans ce travail à identifier les cellules productrices de cette cytokine et les mécanismes de régulation de sa production au cours de la régénération après hépatectomie partielle.

Dans un premier temps, les auteurs [2] ont complété les observations faites par l'équipe de B. Gao [7], qui avait montré que, chez des souris transgéniques surexprimant I'IL-22, la régénération hépatique après hépatectomie partielle (résection chirurgicale des 